\title{
Using Plickers Cooperate with Peer Instruction to Promote Students' Discussion in Introductory Physics Course
}

\author{
Sura Wuttiprom*, Khanti Toeddhanya, Aakapong Buachoom, Karntarat Wuttisela \\ Faculty of Science, Ubon Ratchathani University, Thailand
}

Copyright $\bigcirc 2017$ by authors, all rights reserved. Authors agree that this article remains permanently open access under the terms of the Creative Commons Attribution License 4.0 International License

\begin{abstract}
In decades Peer Instruction (PI) has been confirmed that it can improve students' conceptual understanding. Anyway the main problem for using PI is an audience responding system which required for gathering students' answer, to enhance learning process of PI instead of using Clickers which cost about 40 USD per item. In this work we decided to use Plickers: the free application in smartphones. The purpose of this research is to develop the students' conceptual understanding in work and energy by using PI strategy. The sample for this study came from the different academic year at Ubon Ratchathani University, Thailand. There were 50 first year chemistry students who have enrolled in introductory physics course in academic year of 2016 and 119 first year engineering students who have enrolled in introductory physics course in academic year of 2017. The pre-posted test was the conceptual understanding test in work and energy there were 6 items with 4 choices. The items were a part of the Multiple-choice Test of Energy and Momentum Concepts developed by Chandralekha Singh and David Rosengrant. The pre-posted score had escalated about $13 \%$ (chemistry students) and $25 \%$ (engineering students). Furthermore both groups of students also had a positive attitude to teaching and learning physics with PI and Plickers approach.
\end{abstract}

Keywords Peer Instruction, Plickers, Work and Energy

\section{Introduction}

\subsection{Peer Instruction}

During the past $30-40$ years, the physics education researchers had devoted mentally and physically to develop the researches for solving the problems related to physics learning management. One of the published researches is the Peer Instruction (PI) management developed by Eric Mazur professor at the Department of Physics and Applied Physics, Harvard University [1]. The PI-based learning management is the active learning management for students to understand scientific concepts with the set of questions called ConcepTest through peer discussion. PI-based learning management is the highly effective learning management approach based on the learning assessment framework of Hake [2]. This is because there are some researches confirming that from the PI-based learning management in 30 subjects, there are only 3 subjects which are in low gain. The rest are in the medium gain [3]. In addition, PI-based learning management also enhances the interest / attention on learning management of the students as well [4].

\subsection{The Interactive Management System between the Instructors and the Learners}

Technological advancement enhances the class management during the discussion to be more effective changing from oral answering, hand raising, sign board answering or writing the answers on the paper. It is the classroom response system which can collect data, display results, give feedback, and evaluate learners at the same time [5]. Such system is widely used in leading universities of the world, for example, the University of Edinburgh, Harvard University, etc. PI teaching helps creating the collaboration among the learners in building the knowledge altogether [6]. However, the learning management model applying the classroom response system introduced at earlier stage as the PI learning management is still ineffective [7].

\subsection{Clickers}

The Clickers set (Figure 1) is the set of equipment developed under the audience response systems [8]. It is the set of equipment quite costly (around 1000 - 1500 USD depending on the brand). 1 set of Clicker contains 32 data storage cartridges for 32 students. Therefore, Clicker is not widely available in Thai educational system. However, due to the quick technological advancement at present, the technology is highly efficient, cost-effective and accessible to all classes, for example, the mobile phones which we call 
"smart phone". The educators have developed an interactive management system between instructors and learners on mobile phones called "Plickers" [9].

\subsection{Plickers}

Plickers is the program for the interaction between the mobile phone with cameras installed with the Plickers application for scanning QR-Code (Figure 2). There are 63 sheets of data and all of the answers will be processed and displayed on the instructor's mobile phone (Figure 3) and via the web browser (Figure 4) at the same time.

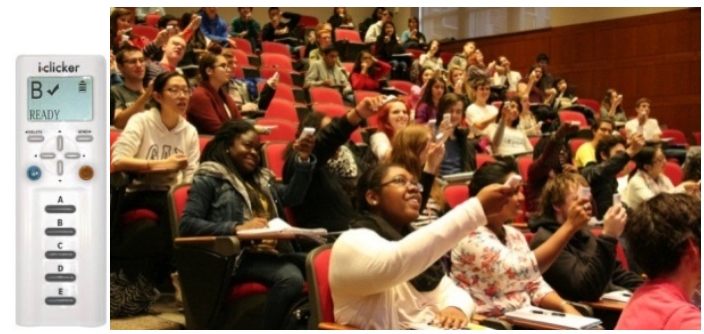

Figure 1. Data storage cartridges and atmosphere of learning with Clicker

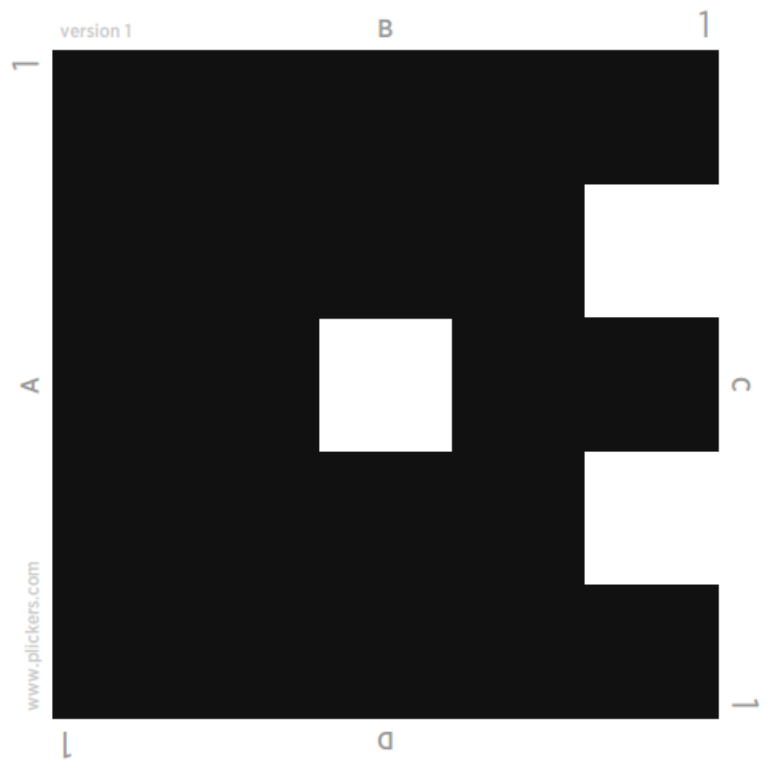

Figure 2. QR-Code sheet to be used with Plickers

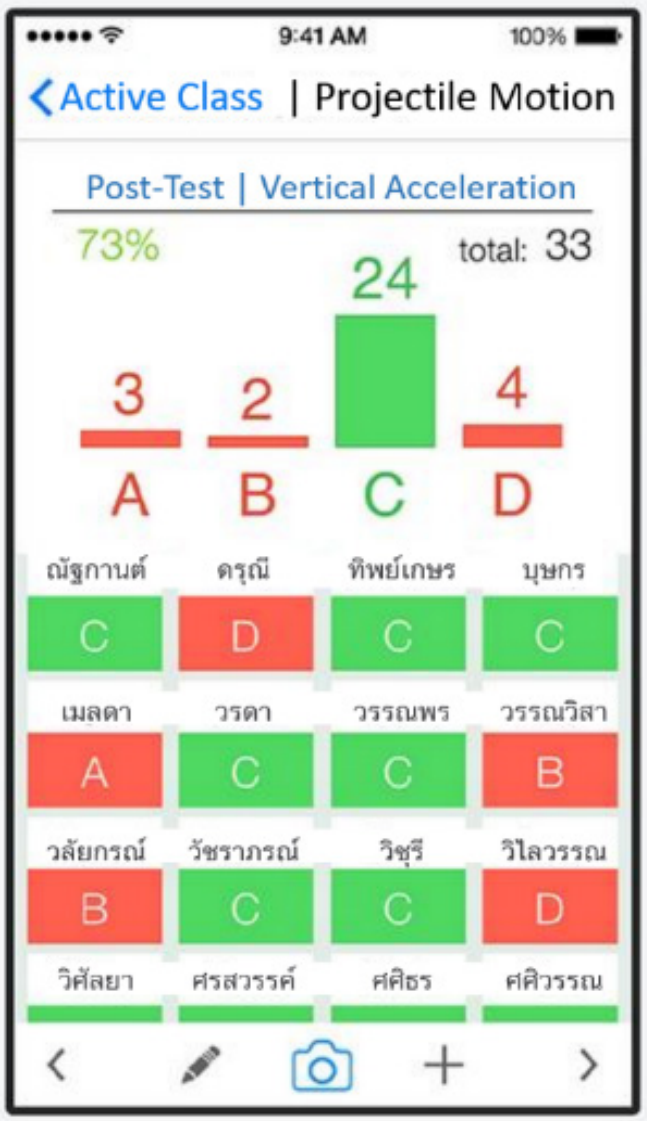

Figure 3. Display of answers of the learners on mobile phone 


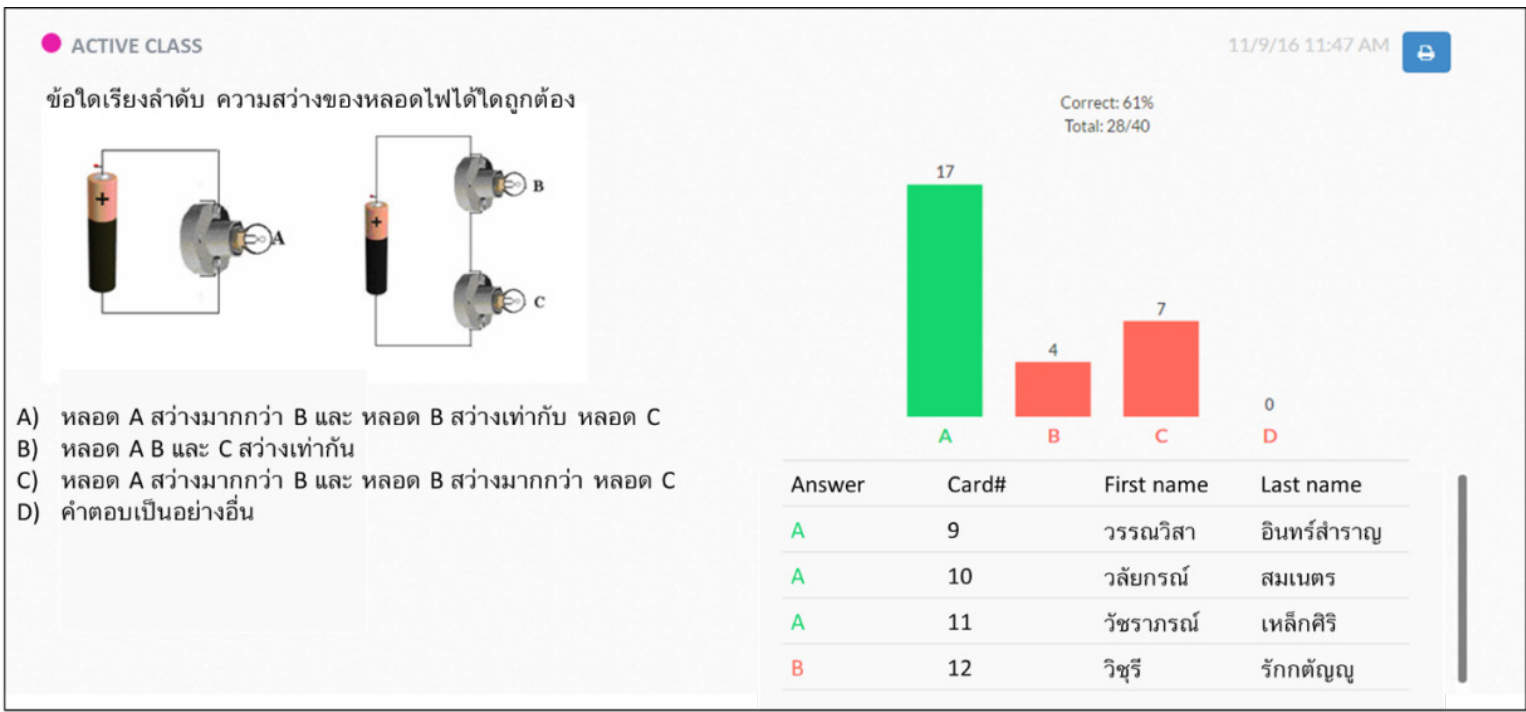

Figure 4. Display of answers of all learners via web browser

\section{Understanding the Scientific Concepts about Energy Work}

The definitions of energy have many different contextual aspects. For example, in some physics books, it defines the energy as the ability to cause work. This is not entirely correct because according to the second item of thermodynamics law, there is some energy that cannot produce work [10]. Moreover, the energy also appears in the fields of biology, chemistry, astronomy, and geology [11, 12]. Thus, explaining about work and energy requires different contexts.

The energy contains five important characteristics [13] which are:

- Energy conservation - In a closed system, energy can neither be created nor destroyed. This is one of several conservation laws used in physics.

- Energy degradation - The total amount of useable energy in a system may decrease over time. This may take the form of energy dissipation (energy lost from an open system) or energy transformation within the system to a less useful form. For example, a cup of hot tea will eventually cool. The thermal energy from the tea has been 'lost' to the surrounding environment. It has not been destroyed, but is no longer a part of the cup and tea system.

- Energy transformation - The energy can be transformed from one form to another, for example, dropping the ball by in gravitational field of the earth, the potential energy can be transformed to kinetic energy.

- Energy transfer - The energy can be transferred between components in a system; in a collision, one billiard ball transfers its kinetic energy to another.

- Energy source - The energy can be added to the system. For example, the Earth is the system. The Earth receives energy from the sun which is very needed to compensate for the heat that the Earth loses outside the atmosphere.

Previous researches have shown that learners still have misunderstanding on the concepts of work and energy. For example, the gravitational energy is fictional [14]. The kinetic energy is vector quantity of work because the gravity is based on the distance in the level. The research of Singh and Rosengrant gives the notice that the reason why the learners have misunderstanding on the concepts of work and energy is partly from the meaning of work in everyday life is different from the meaning of work in physics [15].

\section{Objective}

The researcher found that the PI learning management model has the advantages in the discussions with classmates. If it is used in conjunction with Plickers application that is featured in terms of instant data storage and display, the learners can be developed to have better understanding on the concept of science in that subject or not and what are the attitudes of learners generally towards physics will be. The researcher selects the topic of work and energy which is an important topic for students of all levels and all majors.

\section{Methodologies}

\subsection{Sample}

The sample groups for this study were first year students in bachelor degree of chemistry $(\mathrm{N}=50)$ of academic year 2016 and first year students in bachelor degree of engineering $(\mathrm{N}=119)$ of academic year 2017, Ubon Ratchathani University, Thailand who have enrolled in introductory physics course by purposive sampling. Most 
chemistry students (98\%) did not like physics and though that physics is difficult. The result was from eliciting students wrote their feeling towards physics at the beginning of the class. However, both engineering students and chemistry students did not have much difference in background knowledge of physics.

\subsection{Data Collection}

The researcher collected data by allowing the students to do six items of pre-test on the work and energy covering three key concepts; work, work due to gravity and laws of energy conservation, and energy. The students were given 10 minutes for doing the test. Afterwards, the instructor handled the PI learning management by using the question called ConcepTest as the important tool for discussion among learners before and after the discussion. The instructor asked the students to give the answers, record the answers, then shown answers by using Plickers application. Therefore, the instructor will know the percentage of students answering the question correctly. If the correct answers are less than $50 \%$, the instructor will conduct teaching following the PI learning management process again (as shown in Figure 5). However, the brief presentation will be conducted in different form by using multiple representations such as energy bar graph, images, equations, or etc [16]. If the correct answers are more than $50 \%$, the instructor will summarize such scientific concepts by using student's answers as cases for giving the right explanation. In addition, before and after learning management, the students have responded to the online questionnaire (Google form) about the attitudes towards learning management of introductory physics courses.

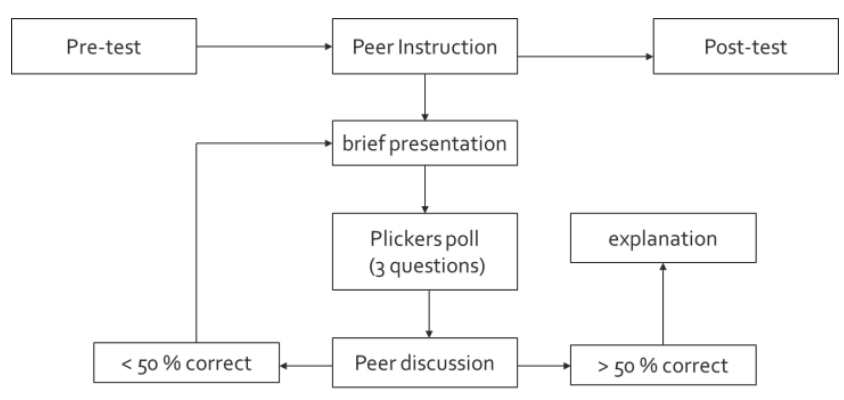

Figure 5. Procedure of data collection

\section{Results and Discussion}

Understanding the scientific concept of work and energy: From the analysis on the average scores of the entire class, it was found that the scores increase from $31.3 \%$ to $46.3 \%$ for chemistry students and $28.3 \%$ to $53.3 \%$ for engineering students. As the result, the scores were lower than $50 \%$ and slightly higher than $50 \%$ for chemistry students and engineering students. When analyzing by using the methods of Hake [1] to evaluate the learning progress, it was found that the normalized gain equaled to 0.22 (chemistry students) and 0.35 (engineering students) in the full proportion of 1.00 which were considered to be low gain $(\mathrm{g}>0.3)$ and the medium gain $(0.3<\mathrm{g}<0.7)$ respectively. The result from chemistry students was different from the research of Chandralekha Singh [16] conducting the PI learning management and finding that learning progress was in the medium gain $(<\mathrm{g}>=0.55)$. However, engineering students' result in this research was in the medium gain but the gain was still lower than the lecture conducted by Chandralekha Singh. Meanwhile conventional learning management had the learning progress in the low gain $(<\mathrm{g}>=0.22)$ and the medium gain $(<\mathrm{g}>=0.35)$. From interviewing both groups of students, they gave the reason that they were not familiar with the kind of understanding questions and they were not confident in answering because in high school, they focused on problem solving (mathematical problem in physics).

When considering the percentage of chemistry students who answered each question correctly in post-test, it was found that there were only three out of six items scored more than 50\% (as shown in Figure 6). In Question 1, the scores of students answering correctly reduced after learning, in contrast the number of engineering students who had scored in this question doubled. Question 1 is about the work due to gravity which depends on altitude only. However, the students still had misunderstandings on the high lifting speed and high lifting characteristics causing the different works due to gravity (Figure 8). Although PI-based learning management in this research has not been successful (the learning progress is in the low gain), such learning management still made the students have post-test scores higher than those of the pre-test in the score range from 40-100 percent as shown in Figure 9. On the other hand, engineering students had a better understanding in overall conceptual tests as shown in Figure 7 and 10. 


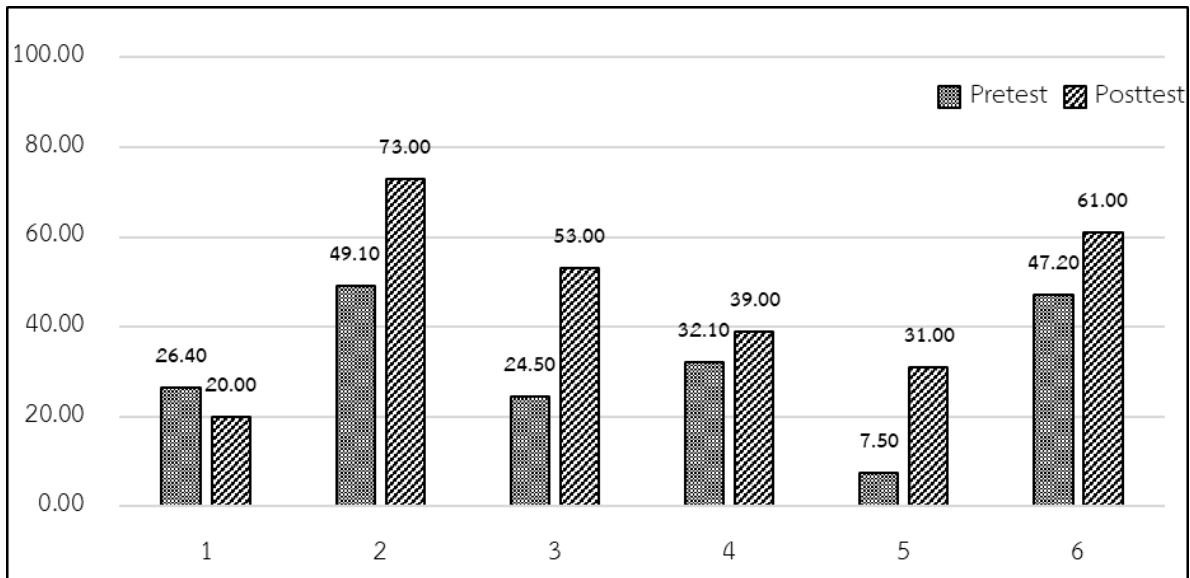

Figure 6. Average scores (percentage) of chemistry students before and after PI-based learning management in each item

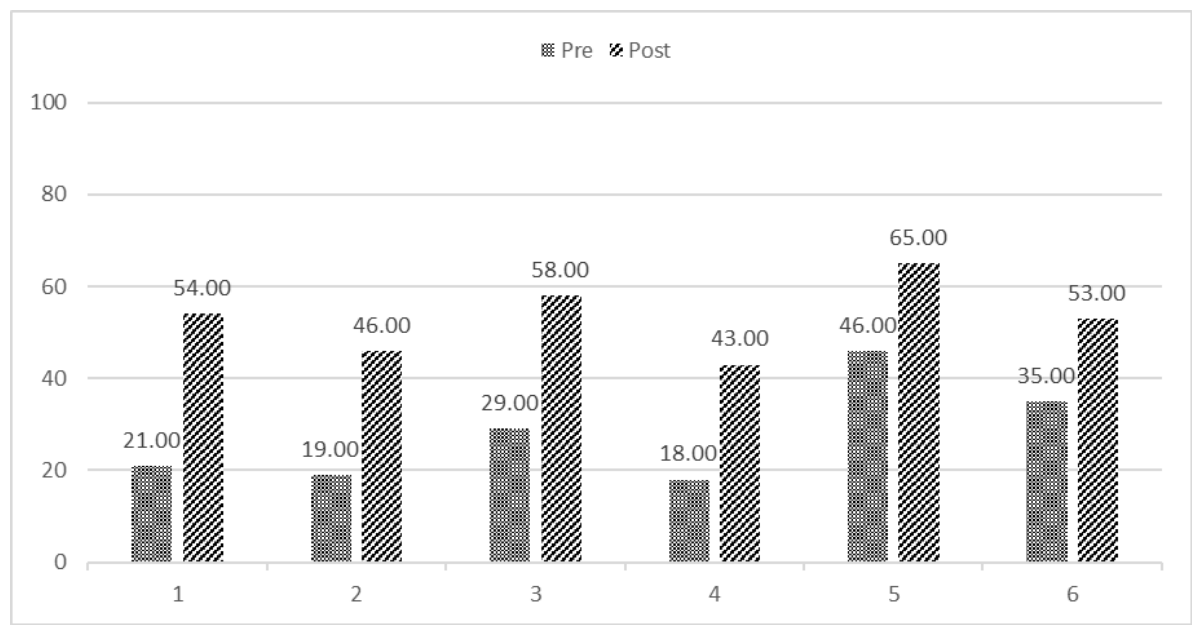

Figure 7. Average scores (percentage) of engineering students before and after PI-based learning management in each item

Question 1 When you lift your luggage from the floor and place it on the table, which one of the followings is correct?

(1) Lifting straight up and carrying it to place on the table cause the different works due to the gravity.

(2) Fast lifting and slow lifting cause different works.

(3) Different heights of tables result in different works.

\begin{tabular}{|c|c|c|c|c|c|}
\hline \multirow[b]{2}{*}{ Choices } & & \multicolumn{2}{|c|}{ Chemistry students } & \multicolumn{2}{|c|}{ Engineering Students } \\
\hline & & Pre-test (\%) & Post-test (\%) & Pre-test (\%) & Post-test ((\%) \\
\hline & (1) only & 11.6 & 40 & 14.3 & 8.4 \\
\hline & (3) only & 26.4 & 20 & 21 & 53.8 \\
\hline & (2) and (3) only & 15 & 8 & 18.5 & 14.3 \\
\hline & (1) and (3) only & 47 & 32 & 46.2 & 23.5 \\
\hline
\end{tabular}

Figure 8. Percentage of students choosing the answer in each item before and after the learning management ( $\mathrm{b}$ is the correct answer) 


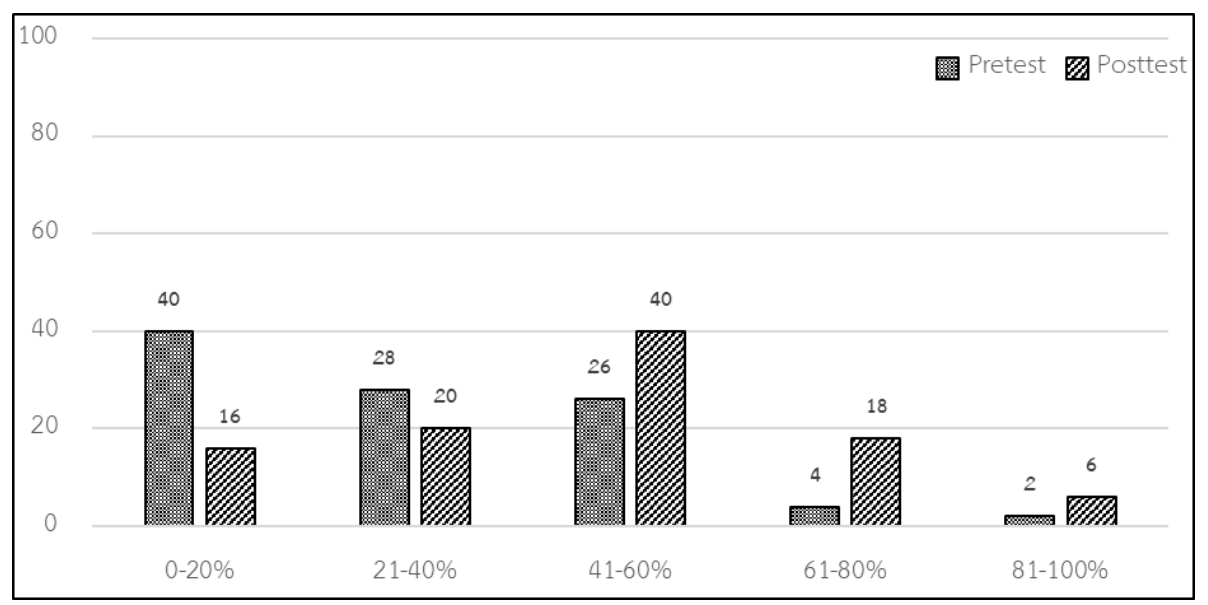

Figure 9. Number of students (percentage) of chemistry students following the score ranges 20 points per range (before and after PI-based learning management)

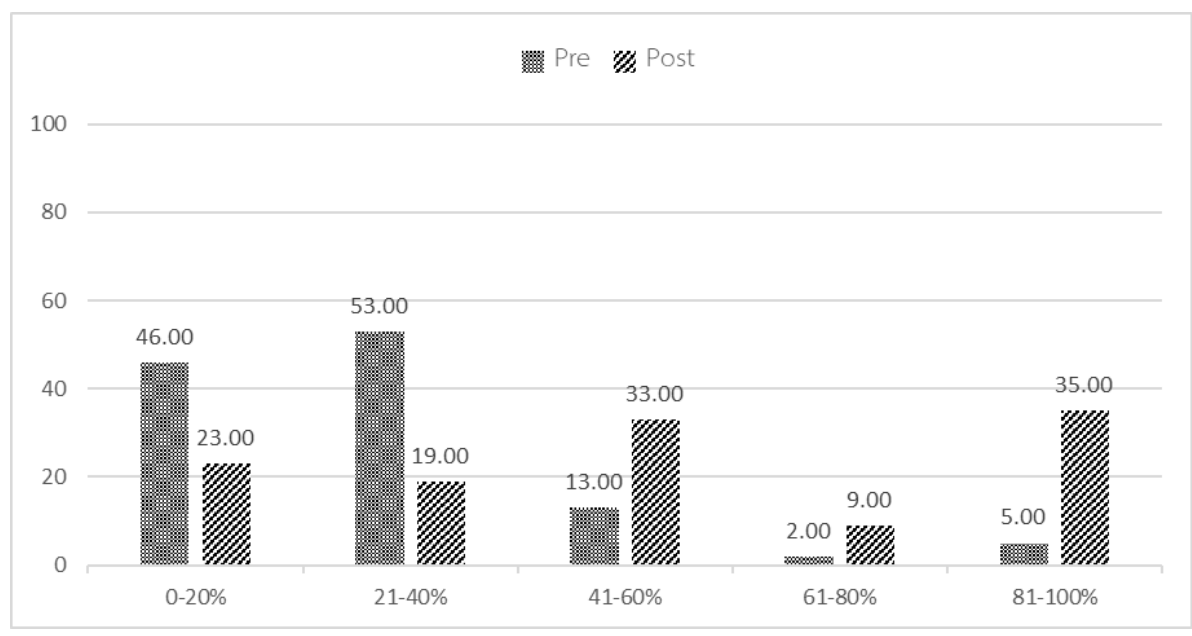

Figure 10. Number of students (percentage) of engineering students following the score ranges 20 points per range (before and after PI-based learning management)

The teacher teaches fun. I like the answering by using technology of the teacher. That the teacher uses mobile phones to scan the answers enjoy us
and make us unstressed. We can also evaluate our understanding as well in order to know which answer we choose and why we choose it. The
students dare to share the ideas without pressure.
It is changed because the teacher always stimulates us and tries to find the experiments related to the contents for us to try and enjoy. Although we do
not totally understand what we study, we want to study because the teacher teaches fun.
Regarding the teaching method, if the students do not understand what they learn, they will become sleepy or the atmosphere in studying will be
boring. However, the teaching method of the teacher is fun. The VDO is used to make us see the picture clearly. We can listen to the music in order
to be relaxed and have less pressure.
The descriptive teaching styles with examples in the class make us see the picture clearly. Playing the game by using the PLICKERS sheets and the
mobile phones to answer the questions on the screen is fun and exciting. It requires both speed and accuracy.

Figure 11. Attitudes of students after the PI learning management

Regarding the attitudes of students before and after learning physics with Plickers in combination with PI learning management before learning management, 98 percent of chemistry students did not like physics and though that physics is difficult. However, after the learning management, the researcher asked the students to write whether their attitudes towards physics have been changed or not and how. Chemistry students were divided into two groups. Group 1 had not changed their attitudes (13\%). That was before learning management, they did not like physics and after the learning management, they still did not like physics. Group 2 had changed their attitudes positively $(87 \%)$. The students said that the Peer Instruction approach, the characteristics of the instructor (good-tempered, fun, kind, no pressure, easily accessible, motivate learners), and technology for learning (Plickers) was the main reasons for the change of attitudes. Samples of texts written by chemistry students were as shown in Figure 11.

From formal interviewing engineering students tended to like physics more. (shift from $73 \%$ to $95 \%$ after lecturing) 
and gave positive feedback. Most of them claimed that they knew that they were not good at physics but they had to work hard on this subject because physics was the essential part in their future career (engineering).

\section{Conclusions}

From analyzing the average scores of chemistry students' score increased from 31.3 percent to 46.3 percent, the average scores were less than half of the full scores. On the other hand, students from faculty of engineer scored slightly lower than chemistry students which were 28.3 percent but at the end of the class they scored 53.3 percent. Even the number of engineering students $(\mathrm{N}=119)$ were higher than the number of chemistry students $(\mathrm{N}=50)$ but the average score of engineering students was higher that because engineering students concerned about physics more than another group. The researcher found that students in both groups were not familiarized to the conceptual understanding tests according to the secondary education has focused on problem solving in order to compete in higher education. Due to the results of analysis on the learning progress using the method of Hake [1], the normalized gain is 0.22 and 0.35 which is in the low and medium level respectively. However, PI learning management by using Plickers to collect data and display result instantly makes the attitude toward learning management in physics change in the good way up to $87 \%$ (chemistry students) and 95\% (engineering students).

\section{Acknowledgements}

This work was supported in part by Ubon Ratchathani University (UBU) and Thailand Center of Excellence in Physics.

\section{REFERENCES}

[1] E. Mazur \& R.C. Hilborn. Peer Instruction: A User's Manual. Physics Today, 50(68), 1997.

[2] R.R. Hake. Interactive-engagement versus traditional methods: A six-thousand-student survey of mechanics test data for introductory physics courses, American journal of Physics, 66(1), 64-74, 1998, doi:10.1119/1.18809.

[3] A.P. Fagen, C.H. Crouch, \& E. Mazur. Peer instruction: Results from a range of classrooms, The Physics Teacher, 40(4), 206-209, 2002, doi:10.1119/1.1474140.

[4] C. Turpen \& N.D. Finkelstein. Not all interactive engagement is the same: Variations in physics professors' implementation of Peer Instruction, Physical Review Special Topics-Physics Education Research, 5(2), 020101, 2009, doi:10.1103/PhysRevSTPER.5.020101.

[5] D. Bruff. Classroom Response Systems ("Clickers"). Vanderbilt Center for Teaching. Retrieved from https://cft.vanderbilt.edu/guides-sub-pages/clickers, 2017.

[6] C. Singh \& D. Rosengrant. Multiple-choice test of energy and momentum concepts, American Journal of Physics, 71(6), 607-617, 2003, doi:10.1119/1.1571832.

[7] S. Chalermchat \& S. Wuttiprom. Effect of Peer Instruction on Pattern Analysis of Grade-10 Students' Conceptual Understanding about Force and Motion, J. Res. Unit Sci. Technol. Environ. Learning, 6(2), 232-242, 2015.

[8] J.E. Caldwell. Clickers in the large classroom: Current research and best-practice tips, CBE-Life Sciences Education, 6 (1), 9-20, 2007, doi:10.1187/cbe.06-12-0205.

[9] Amy, N., \& Kim, J. (2016). Plickers.

[10] I.M. Sefton. Understanding energy. In Proceedings of 11th biennial science teachers' Workshop (pp. 17-18), 2004.

[11] J.W. Jewett. Energy and the confused student I: Work. The Physics Teacher, 46(1), 38-43, 2008, doi:10.1119/1.2823999

[12] J.W. Jewett. Energy and the confused student II: Systems. The Physics Teacher, 46(2), 81-86, 2008, doi:10.1119/1.2834527.

[13] R.A. Lancor. Using student-generated analogies to investigate conceptions of energy: a multidisciplinary study. International Journal of Science Education, 36(1), 1-23, 2014, doi:10.1080/09500693.2012.714512

[14] G. Swackhamer \& D. Hestenes. An energy concept inventory. Arizona State University, 2005.

[15] C. Singh. Impact of peer interaction on conceptual test performance. American journal of physics, 73(5), 446-451, 2005, doi:10.1119/1.1858450.

[16] D. Rosengerant, E. Etkina \& A. Van Heuvelen. An overview of recent research on multiple representations. AIP Conference Proceedings, 883(1), 149-152, 2007, doi: http://dx.doi.org/10.1063/1.2508714. 